\title{
EXPERIMENTS OF NANOMETER SPOT SIZE MONITOR AT FFTB USING LASER INTERFEROMETRY
}

\author{
T. Shintake, K. Oide, and N. Yamamoto \\ KEK: National Laboratory for High Energy Physics, Oho, Tsukuba, Ibaraki 305 Japan \\ A. Hayakawa, and Y. Ozaki \\ KHI : Kawasaki Heavy Ind. Ltd., Akashi, 673 Japan \\ D. Burke, R. C. Field, S. Hartman, R. Iverson, P. Tenenbaum, and D. Walz \\ SLAC: Stanford Linear Accelerator Center, Stanford CA 94309, USA
}

The nanometer spot size monitor based on the laser interferometry has been developed and installed in the final focus test beam (FFTB) line at SLAC. The beam experiments started in September 1993, the first fringe pattern from the monitor was observed in the beginning of April 1994, then the small vertical spot around $70 \mathrm{~nm}$ was observed in May 1994. The spot size monitor has been routinely used for tuning the beam optics in FFTB. Basic principle of this monitor has been well proved, and its high performance as a precise beam monitor in nanometer range has been demonstrated.

\section{INTRODUCTION}

In order to prove the feasibility of $\mathrm{TeV}$-scale electronpositron linear colliders, the FFTB beam line has been constructed at SLAC under the international collaborations[1]. This specially designed focusing system aims to focus a low-emittance electron beam to a tiny flat beam of $1 \mu \mathrm{m}$ in horizontal and $60 \mathrm{~nm}$ in vertical sizes. To achieve the design spot of FFTB, many new instrumentations have been developed and applied[2].

Among these developments, measurement of the extremely small spot size is one of challenges in this project. In 1990, a new method was devised by the author which utilizes Compton scattering of photons in a laser interference fringe[3]. Figure 1 shows schematic diagram of this monitor. A laser beam is split into two beams and folded onto itself to produce an interference fringe pattern in space. The electron beam is scanned across this pattern to yield a modulated rate of Compton-scattered photon in the forward direction. The depth of the modulation corresponds to the spot size.

The spot size monitor system was developed by KEK and $\mathrm{KHI}$, and installed in the focal point of FFTB line in August 1993[4]. This monitor has six laser beam lines in one interferometer table. By choosing a set of two laser beam lines, this monitor can produce three different measuring ranges: $0.8 \mu \mathrm{m}<\sigma_{\mathrm{x}}<4 \mu \mathrm{m}, 150 \mathrm{~nm}<\sigma_{\mathrm{y}}<750$ $\mathrm{nm}$, and $40 \mathrm{~nm}<\sigma_{\mathrm{y}}<200 \mathrm{~nm}$.

The monitor has been commissioned in April 1993, and routinely used for the beam tuning. In the beam operation, we found several problems, such as, low signal to noise ratio of $\gamma$-ray, laser bean position drift, laser pulse timing drift, and laser beam position pulse-to-pulse jitter. To settle these problems, we improved the hardware and also software in this system. In this paper, practical operation method, example results, and also the hardware improvements are described.

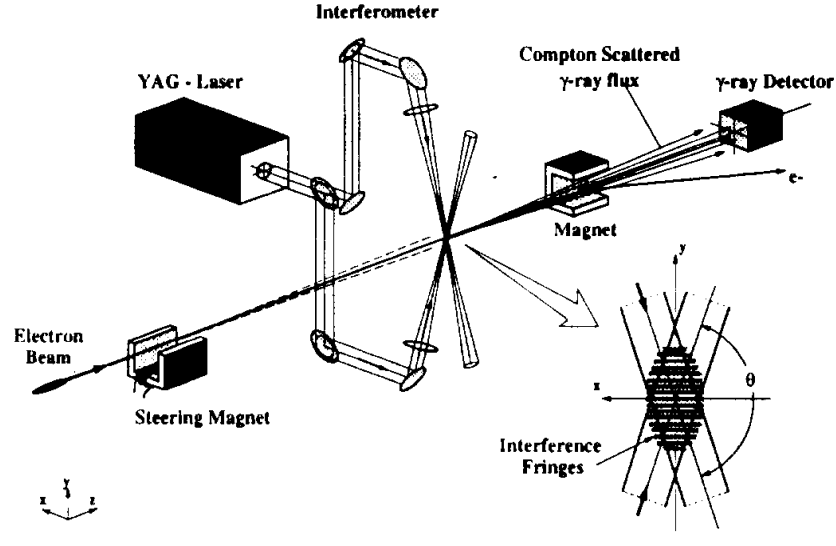

Fig.1 Schematic diagram of the spot size monitor using laser interferometry.

\section{MEASUREMENT PROCEDURE}

\section{A. z-axis alignment}

For $z$-axis alignment of the laser beams, we prepared a slit scanner. We insert the slit of $0.5 \mathrm{~mm}$ width at the focal point, and scan the z-position of laser beam by the mirror mover. We measure the transmitted laser power through the slit using a photo-detector at opposite side. From this measurement, we can determine the center of the slit looking from laser beam and set the mirror position to this center. By repeating this process for all laser beam lines, we can align all laser beam lines in one plane.

\section{B. $x y$-axis alignment}

For $x y$-axis alignment, we use $\gamma$-ray signal from the electron beam. By scanning the electron beam trajectory along $x$ or $y$ axis, we look for a $\gamma$-ray peak of the laser beam. By adjusting $x$ or $y$ axis of each mirror, we can align each laser beam to the center( electron beam axis ).

\section{Q-switch timing adjustment}

The laser has pulse length of $10 \mathrm{nsec}$. We have to adjust the laser pulse timing just on the electron beam timing. To do this, we measure the $\gamma$-ray signal from one laser beam by scanning the Q-switch timing to find out the laser peak.

Since we use a seeding laser in YAG-laser system, the timing of laser peak is sensitive to temperature variation in laser system ( because the oscillator axis is sensitive to 
temperature change). We are preparing a thermocontroller to keep the air temperature within $\pm 0.5 \mathrm{deg}$. C.

\section{Spot Size Measurement}

After the laser beam alignment, we choose fringe type ( by choosing a set of two beam lines from the six beam lines ), and scan the electron beam across the fringe pattern. We take 18 shot data in one point, in which 6 data are Laser_ON data, and 12 data are Laser_OFF data, we subtract the averaged Laser_OFF data from the averaged Laser_ON data to eliminate the background baseline shift.

\section{MEASUREMENT EXAMPLE}

Scanning the electron beam trajectory with fine step, we observe a periodic intensity modulation in the $\gamma$-ray data as shown in Fig. 2(a). The vertical axis is the averaged and noise-subtracted $\gamma$-ray counts (ADC counts ). The solid curve is a least-mean-square fit of analytical function:

$$
\mathrm{Y}=\mathrm{A}+\mathrm{B} \sin \left(\frac{2 \pi}{d} y+\mathrm{C}\right)
$$

where $d$ is a period of the fringe pattern( dark-to-dark distance ). We use theoretical value of $d=\lambda_{0} / 2 \sin (\theta / 2)$, where $\lambda_{0}$ is wavelength of laser, $\theta$ is crossing angle of laser beams, in this case, $\lambda_{0}=1064 \mathrm{~nm}, \theta=174 \mathrm{deg}$., and $d=533 \mathrm{~nm}$. From the fitting, $\mathrm{A}=106.7, \mathrm{~B}=72.6$, and the modulation depth is $B / A=0.68$. From this we calculate the spot size as follows.

Assuming a Gaussian beam, the modulation depth is related to the spot size:

$$
M=B / A=|\cos \theta| \cdot C_{p} \cdot C_{\beta} \cdot \exp \left(-2 k_{y}^{2} \sigma_{y}{ }^{2}\right)
$$

where $|\cos \theta|$ is a correction factor for a traveling wave component in the fringe. For $\theta=174 \mathrm{deg}$, it becomes 0.9945. $C_{p}$ is a correction factor for power imbalance of two laser beams:

$$
C_{p}=\frac{2 \sqrt{P_{2} / P_{1}}}{1+P_{2} / P_{1}}
$$

In the present case, the power ratio $P_{2} / P_{1}$ is 1.26 , and the correction factor is 0.993 . $C_{\beta}$ is a correction factor for laser beam depth in $\mathrm{z}$-direction:

$$
C_{\beta}=\frac{1}{\sqrt{1+\left(k_{y} \sigma_{y}^{*} w_{0} / \beta^{*}\right)^{2}}}
$$

where $k_{\mathrm{y}}$ is the wave number of laser beam in $y$-direction:

$$
k_{y}=k_{0} \cdot \sin (\theta / 2)=2 \pi / \lambda \cdot \sin (\theta / 2)=5.90 \mu \mathrm{m}^{-1} .
$$

$w_{0}$ is a laser waist size at the focal point. Sine this correction factor is also a function of the spot size, eq. (2) can not be simply solved. Because of this reason, in a first step, letting $C_{\beta}=1.0$, we calculate a raw spot size from eq.(2). In this example, the spot size is $\sigma_{y^{*}}=73.2$ nm. Figure 2 (b) shows the distribution of the measured raw spot size in the last 3 hors of the run in May 1994. The distribution shows that the beam line of FFTB has the stability of $\sigma_{y}{ }^{*}=70 \pm 7 \mathrm{~nm}$.
For the $70 \mathrm{~nm}$ spot size, the correction factor $C_{\beta}$ for laser beam depth in z-direction becomes 0.92 , where we put $w_{0}=100 \mu \mathrm{m}, \beta^{*}=100 \mu \mathrm{m}$. With this correction, the spot size in Fig. 2 (b) becomes $\sigma_{y}^{*}=65 \pm 7 \mathrm{~nm}$.
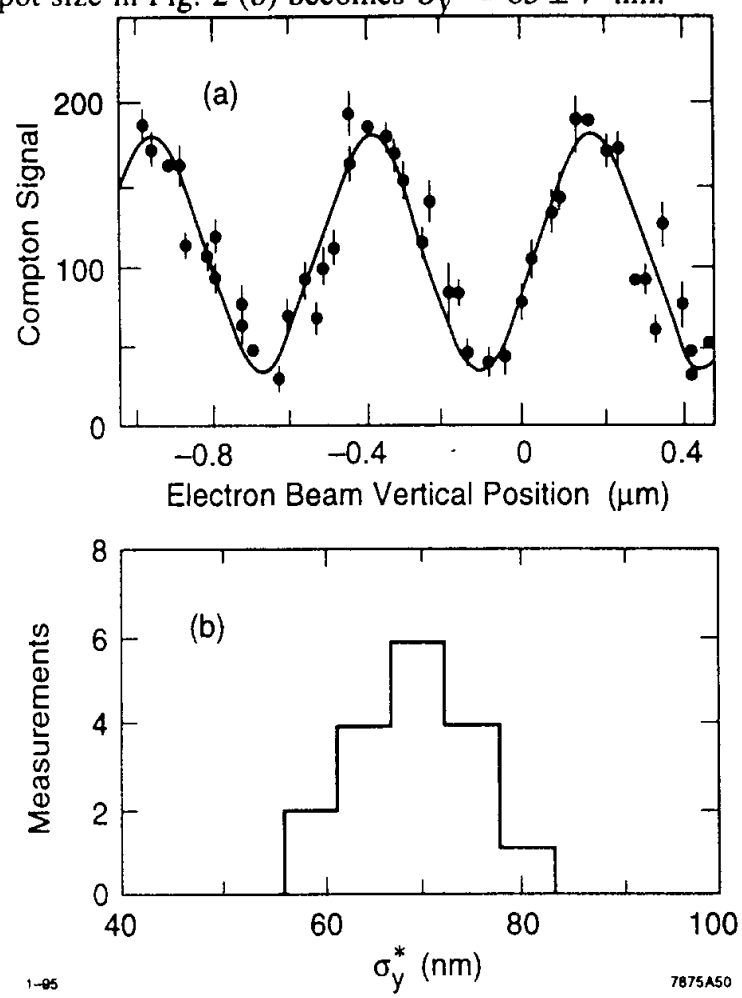

Fig. 2. (a) Measured fringe pattern in the Compton scattered $\gamma$-ray. Solid curve is the least-square-fit of sinfunction. (b) Spot size distribution for 3 hours measurement.

\section{HARDWARE IMPROVEMENTS}

Figure 2 (b) shows the spot size distribution of the measured in the last 3 hours of the run in May 1994. This distribution shows that the beam line of FFTB has the stability of $70 \pm 7 \mathrm{~nm}$. However, the stability includes possible fluctuations in the spot size monitor. In order to resolve the electron beam stability from the spot size monitor stability, we started improvement work right after the run. Some of the improvement has been done before the September run in 1994, and tested. They are summarized bellow.

\section{A. YAG-Laser}

We use pulsed Nd: YAG-laser, which emits Q-switched pulse light at $1064 \mathrm{~nm}$ of infrared wavelength, pulse length $10 \mathrm{~nm}$, pulse energy of $200 \mathrm{~mJ}$ and repetition rate at $10 \mathrm{pps}$. A seeding laser is implemented to keep the coherency better than $3 \mathrm{~m}$, which is quite enough to our measurement. We always monitor the laser light timing to make sure the pulsed laser light is on timing to the electron bunch. With this monitoring, also we can contirm that the laser is fully locked to the seeding source (in case of out-of-locking, the timing shift back 30 nsec ). The unwanted circular polarization component, and the perpendicular component are eliminated by means of high power polarizers. 
In the September-1993 run, we observed a discrepancy in measured IP spot size. One of the possible error sources is beam pointing pulse-to-pulse jitter of laser beam. This phenomena is related to (a) fluctuation of stored energy of the seeding laser, (b) jitter of the flashlamp pumping power, (c) cavity-axis fluctuation in the oscillator due to mechanical vibration, and (d) unwanted mirror tilt due to piezo mover in seeding system. Inspection of these items and improvements are anticipated for the next run.

\section{B. Laser Beam Transport System}

In order to avoid beam position fluctuation due to unstable refraction in the air, the laser beam is transported in vacuum about 30 meters from the laser building to the interferometer inside the FFTB tunnel. The YAG-laser has a big pointing drift ( $0.5 \mathrm{mrad} \max$.) due to temperature change in cooling water of YAG-rod and ambient air temperature change. In order to eliminate the beam position movement, a feedback loop is implemented, which keeps the laser beam position at the entrance on the interferometer to the center by controlling the mirror angle in front of the YAG-laser

However, in the September-1993 run, it was found that a mirror in the transport line was moving due to the sun light heating the concrete roof of FFTB tunnel, and the laser beam position at the focal-point was always moving around about a few tens of $\mu \mathrm{m}$. In the spot size measurements, we frequently needed to check the position and trim the final mirrors.

In order to eliminate this drift, another feedback loop has been implemented. In the present system, one feedback loop keeps the incoming laser beam to the center at the interferometer entrance, and the second loop keeps the focused beam at IP to the center. In the recent beam run at March-1995, the stability has been quite improved. no beam position drift was observed.

\section{Noise Background Subtraction}

Since the spot size is determined by the modulation depth in the Compton scattered $\gamma$-ray data, i.e., the ratio of AC to DC components, for accurate measurements it is very important to eliminate the effect of the noise background. To do this, a synchronous detection technique is used. When the electron beam runs at $30 \mathrm{~Hz}$, the laser is fired at $10 \mathrm{~Hz}$. That is, for each electron beam pulse the laser beam is fired alternatively: ON_OFF_OFF_ON_OFF_OFF... The laser-ON $\gamma$-ray data is averaged for six pulses, and the noise-background ( laser-OFF) $\gamma$-ray data is averaged for twelve pulses, then the noise-background data is subtracted from the laser-ON data.

\section{Interferometer Optics}

In order to measure the spot size accurately, we have to overlap two laser beams precisely at the focal point. If the laser beam diameter at the focal point is larger, the alignment tolerance becomes looser. However, if the laser beam diameter is larger than the $\beta$-function at IP, it becomes difficult to directly measure the minimum spot size at electron beam waist. Also the $\gamma$-ray count per pulse becomes lower, which is inversely proportional to the laser beam diameter. On the other hand, if the laser beam diameter becomes smaller, we can get good $\gamma$-ray count and we can measure the spot size with good S/N ratio. However, the alignment tolerance becomes severe.

In the initial design, we chose the laser beam diameter to the upper limit, that is, $\sigma_{\mathrm{L}}=50 \mu \mathrm{m}$. The alignment was easy, but the $S / N$ ratio was as low as $3: 1$. We changed the laser beam optics, and made the beam size smaller: $\sigma_{\mathrm{L}}=30 \mu \mathrm{m}$, then the $\mathrm{S} / \mathrm{N}$ ratio was improved twice.

\section{SUMMARY}

Nanometer beam was produced in FFTB and whose transverse dimensions were successfully measured by the spot size monitor based on the laser interferometry. This success is a big milestone toward the electron-positron linear collider $R \& D$ project. In an actual linear collider, we use a short wavelength laser such as 5th harmonic radiation of Nd: YAG-laser at $213 \mathrm{~nm}$. Fully utilizing the synchronous-noise-background-subtraction technique and using a stable laser oscillator, it will be possible to measure the modulation amplitude up to $95 \%$ or more. The spot size of $3-4 \mathrm{~nm}$ at interaction point can be measured with enough accuracy based on this method.

\section{ACKNOWLEDGMENTS}

We gratefully acknowledge the additional members of FFTB collaboration and all who contributed to this project, whose various support was key to its success.

\section{REFERENCES}

[1] M. Berndt et al.,"Final Focus Test Beam Design Report, SLAC-REF-376(1991).

[2] V. A. Alexandrof, et al.., "Results of Final Focus Test Beam", $n$ these proceedings.

[3] T. Shintake, "Proposal of a nanometer beam size monitor for $\mathrm{e}^{+} \mathrm{e}^{-}$linear colliders", Nucl. Instrum. and Meth. in Physics Research A311 (1992) 453-464.

[4] T. Shintake, H. Hayano, A. Hayakawa, Y. Ozaki, M. Ohashi, K. Yasuda, D. Walz, S. Wagner and D. Burke, "Design of Laser-Compton Spot Size Monitor", proc. XVth Int. Conf. on High Energy Accelerators, Hamburg, Germany July 1992, pp. 215-217

[5] V. Balakin et al., "Focusing of Submicron Beams for TeV-Scale e+e- Linear Colliders", Phys. Rev. Let. Vol. 74, 27 March 1995, pp. 2479-2492 